\title{
Clinical Outcomes and Donor Site Morbidity of Anterior Cruciate Ligament Reconstruction with Full Thickness Peroneus Longus Tendon
}

\section{Zhe Ge ( 763144116@qq.com )}

Department of Orthopaedics, Jinshan Hospital, Fudan University

\section{Bingnan Wang}

Department of Orthopaedics, Jinshan Hospital, Fudan University

\section{Xinchao Zhang}

Department of Orthopaedics, Jinshan Hospital, Fudan University

Shichao Zhang

Department of Orthopaedics, Jinshan Hospital, Fudan University

\section{Research Article}

Keywords: Anterior Cruciate Ligament Injury, Peroneal Longus Tendon, Arthroscopy, Ligament Reconstruction

Posted Date: January 4th, 2021

DOI: https://doi.org/10.21203/rs.3.rs-127706/v1

License: (c) (i) This work is licensed under a Creative Commons Attribution 4.0 International License. Read Full License 


\section{Abstract}

Backgroud: Anterior cruciate ligament $(\mathrm{ACL})$ is an important anatomical structure for maintaining the stability and moveability of a knee joint. When the load exceeds its strength, ACL will be damaged or broken. The main therapy for a fractured $A C L$ is the arthroscopic ligament reconstruction technique. Commonly used reconstruction materials are grouped into three categories: autologous tendons, allogeneic tendons and artificial materials. Peroneal longus tendon(PLT) is an autograft, its superficial location and easier access contribute to its increasing application as a new graft substitute in cruciate ligament reconstructions over recent years. Our research is aiming to investigate the functional recovery of the knee joint after anterior cruciate ligament transplantation with autologous peroneus longus tendon under arthroscopy and its influence on the ankle joint of the donor site.

Methods: During the research period of our retrospective analysis from January 2015 to July 2019 , the single-bundle double-stranded anatomical reconstruction technique was applied under arthroscopy on 32 patients with acute anterior cruciate ligament ruptures (consisting of 22 males and 10 females in the 1645 (31.1 \pm 8.6) age bracket, and among them, 18 cases suffered knee injury on the left limb and the other 14 on the right one) within three weeks of their injury, and the graft for the anterior cruciate ligament (fixed with an Endobutton plate on one end and a screw on the other) was the autologous peroneus longus tendon on the ipsilateral limb. The IKDC score, KOOS score and Lysholm score were used to evaluate the functionality of the affected knee joints and the AOFAS score that of the tendons and ankle joints.

Results: All patients were followed up for 1 year (14.5 \pm 1.6 months). One case developed postoperative knee joint infection but recovered soon following active treatments, and all the patients turned negative in the postoperative Lachman tests and pivot-shift tests. At the last follow-up, IKDC score, KOOS symptom score, KOOS pain score, KOOS functional life score, KOOS sports entertainment score, KOOS life quality score and Lysholm score were $82.1 \pm 13.5,90.8 \pm 15.5,90.4 \pm 5.9,97.8 \pm 4.3,73.7 \pm 26,83.9 \pm 18.1$ and $87.3 \pm 12.0$, respectively, showing marked improvements on and statistically significant differences $(P<$ $0.05)$ from the preoperative figures. The AOFAS score of the affected ankle joints was $94.7 \pm 10.8$, signifying a good functional recovery. MRI observation one year later showed that the reconstructed ligament had extended continuously to the position satisfying the requirements of anatomical reduction, and the bone tunnels and tendons had healed well.

Conclusion: Reconstruction of the anterior cruciate ligament with a single-bundle of double-stranded peroneus longus tendon can satisfactorily restore the function of an injured knee joint without a significant impact on the donor ankle joint.

\section{Backgroud}

Anterior cruciate ligament (ACL) is an important anatomical structure for maintaining the stability and moveability of a knee joint ${ }^{[1]}$, and the lengths of its collagen and elastic fibers will change with the stress 
in it under a physiological load. When the load exceeds its strength, ACL will be damaged or broken, not only reducing the stability and moveability of the knee joint but also accelerating its degeneration ${ }^{[2]}$. The main therapy for a fractured $\mathrm{ACL}$ is the arthroscopic ligament reconstruction technique, and ideal materials for such a reconstruction should have a sufficient length and diameter, perform physiological functions similar to those of the original ACL, including strength, toughness and tension curves, and have no significant adverse effects on the donor site. Commonly used materials are grouped into three categories: autologous tendons, allogeneic tendons and artificial materials. Notably, autologous hamstring tendon (Hamstring tendon, $\mathrm{HT}$ ), bone-patellar tendon-bone, etc. have been maturely used in the cruciate ligament reconstruction surgery. Yet, all the alternatives mentioned-above have their respective disadvantages.

Peroneus longus tendon (PLT) has been proven by biomechanical experiments to have similar biomechanical properties to cruciate ligaments, and its superficial location and easier access also contribute to its increasing application as a new graft substitute in cruciate ligament reconstructions over recent years ${ }^{[3-5]}$. However, its impacts on the functions of the donor ankle joint after transplantation are scantily reported due to a shorter application history. In order to further explore the feasibility of peroneal longus tendon as a graft, we retrospectively analyze the clinical data of patients who underwent arthroscopic ACL reconstruction with autologous PLT from January 2015 to July 2019 in our hospital. The reports are as follows:

\section{Materials And Methods}

\section{Basic facts}

The group includes 20 male patients and 12 female ones in the age bracket of $16-45$ years old (31.1 \pm 8.6$)$. All the cases are acute injuries, with 18 injured on the left knee and 14 on the right knee, and their hospital stay lasted 5-23 days (13.6 \pm 5.8$)$. There were various causes behind injuries: 10 cases were injured in traffic accidents, 10 were injured by falls, 7 were sprained from sports, and 5 were injured by heavy objects.

Inclusion Criteria: confirmed ACL injury (alone or concurrent with meniscus injury; Fig1), aged 16-45 years old and having complete follow-up data available. Exclusion Criteria: concurrent with a ruptured posterior cruciate ligament, medial and lateral collateral ligament or patellar support belt, injured cartilage tissues, fractures around the knee joint, and/or a diseased lower limb and/or an abnormal contralateral knee joint; more than 1 year after the surgery; concurrent with severe skeletal, muscular or neurological diseases on the ipsilateral limb, such as osteoarthritis, femoral head necrosis, tuberculosis, lumbar disc herniation, diabetic foot,etc; and the history of cerebral infarction and/or abnormal strength of lower limb muscles.

\section{Surgical Method}

All operations were performed by the same surgeon, and the endobutton and BioRCl interface screws from Smith \& Nephew were used as the locking screws in these operations. The patients under general 
anesthesia were lying on the supine position, with balloon tourniquets wrapping around the upper third of their thighs, and the anterolateral and anteromedial approaches for arthroscopic surgery were established. The arthroscopy was then inserted from the anterolateral entrance and careful checks were made to confirm ACL raptures.

PLT preparation: firstly, make an about-3-cm-long longitudinal incision on the affected limb at the position $1 \mathrm{~cm}$ away behind and 2-3 cm over the lateral malleolus (the incision should not be too far away to prevent the possible friction between the incision scar and the upper shoe edge in future) for exposing and separating the PLT and the PBT in order; next, cut off the distal end of the PLT; then, carefully peel off the proximal end of the PLT with a key extractor; further then, cut off the proximal end at the point $4-5 \mathrm{~cm}$ away from the fibular head and weave the removed PLT into a double bundle; and finally, under arthroscopy, strip the remaining ACL off from the synovial membranes and fatty tissues at the femoral attachment point, clean off its stump and keep it. Do be careful all the way to prevent any damage to the common peroneal nerve.

Tibial Tract Fabrication: the tibial tract is about $45-50 \mathrm{~mm}$ in length (consisting of the lengths of the graft and the femoral tract and the intra-articular distance), about $8 \mathrm{~mm}$ in diameter and opens at the intersection of the extension line of the lateral meniscus and the intercondylar ridge, about $7 \mathrm{~mm}$ away in front of the PCL.

Correctly adjust the position and angle of the positioner, insert the guide needle, confirm that the entry point is at the right position, and select a suitable drill for fabricating the tibial osseous canal. The femoral bone canal is fabricated in the following process: firstly, have the knee joint flexed at least 70 degrees, insert the guide wire backward through the tibial canal deeply till reaching the marked opening on the femoral osseous canal (located inferoposterior to the angulation point between the resident ridge a bony landmark for arthroscopic femur localization - and the interfacial ridge); next, have the drill bit pass through the guide needle and the tibial bone canal till touching the back of the femoral wall, with the penetration depth of the drill bit being about 25-30 mm; then, lead the prepared tendon out through the bone canals of the tibia and femur with a traction line, and fix the femur with a looped plate and the tibia with a bioabsorbable screw; further then, check the position and stability of the graft under arthroscopy, and conduct drawer tests while flexing and extending the joint; and finally, suture the incision after confirming no dysfunctions, apply elastic bandage on the incision and support the limb with a brace. After the operation, perform MRI and CT three-dimensional reconstruction examination on the patient's surgical site. (Fig2-3)

\section{Postoperative Treatment and Rehabilitation}

Keep the postoperative knee joint in the compression bandaging for five days for reducing the swelling and fully extended and fixed with a brace for 4 weeks; do isometric exercises of quadriceps and exercises of straight leg lifting 2 weeks later; do protective ROM exercises under partial load and in the range of motion from 0 to 90 degree 4 weeks later; do ROM exercises under full load and in the range of motion from 90 degrees to full extension 8 weeks later; perform daily activities, stand on one leg or do exercises 
with a stationary bicycle 5 months later; do light-intensity physical exercises 6 months later; do continuous jump tests 12 months later; and resume normal physical exercises (if the continuous jump tests show a satisfactory results).

\section{Evaluation Indicators and Statistical Methods}

IKDC score, KOOS score, Lysholm score were used for evaluating the knee joint function of the affected side, AOFAS score for evaluating the ankle joint function of the donor site, and SPSS 20 statistical software for analyzing the collected data of each group in the form of mean \pm standard deviation. First, check the normality of data of each group through Shapiro-Wilk tests, finding that the scores of each group before and one year after the operation did not conform to the normal distribution $(P<0.1)$; then, check the normality of the difference between the data of each group before and after the operation, finding that the difference conformed to the normal distribution ( $P>0.1)$; and finally, compare and analyze the scores before and after the operation through paired $t$ tests, with the test level being $a=0.05$.

\section{Results}

The follow-up period with all the 32 patients varied in the 16-24 months range, with an average of 21.5 months. Their IKDC, KOOS, and Lysholm scores one year later were significantly different from those before surgery $(P<0.005)$, and especially, their ankle AOFAS scores were rather satisfactory, with related results shown in Tables 1, 2 and 3.

One patient suffered a superficial tibial tunnel infection and recovered following active anti-infection and superficial debridement treatments. The postoperative patient turned out negative in the anterior drawer test and the axis shift test, and magnetic resonance re-examination showed that the graft had a good continuity

\section{Discussion}

This research aims to evaluate the clinical and imaging results of PLT reconstruction of the anterior cruciate ligament, and identify any effects of PLT use on the functions of the ankle joint. In our research, ACL anatomical reconstruction with PLT showed satisfactory clinical and imaging results. One year later, the MRI of all the patients showed that their grafts were continuous and their knee joint functions recovered well. Most of the patients could perform sports activities, such as jogging. Hence, there are no significant effects on the ankle functions.

ACL injury has an incidence rate of about 38 cases per 100,000 persons ${ }^{[6]}$, and is mostly seen in: sports activities, such as basketball, football, skiing and other strenuous scenarios, and traumas, such as traffic accidents and fall accidents. At present, the preferred therapy for ACL ruptures is surgical reconstruction to restore the knee stability and reduce the risk of secondary meniscal tears and symptomatic

osteoarthritis ${ }^{[7,8]}$, and the commonly used cruciate ligament reconstruction materials include autologous tendons, allogeneic tendons and artificial materials. In particular, autogenous tendons are widely used in 
the reconstruction therapy in that they not only meet the functional requirements more satisfactorily but also avoids the possible rejection of allogeneic tendons ${ }^{[9]}$. Autologous hamstring tendon, bone-patellar tendon-bone, quadriceps tendon, etc. have been maturely used in cruciate ligament reconstruction surgeries ${ }^{[10]}$. However, various alternatives also have their own disadvantages. For example, an autogenous hamstring tendon has good strength but a uncertain diameter. With the tendon removed, the flexural property and internal rotation muscle strength of a knee joint will weaken, risking the saphenous nerve damage and hamstring muscle strength weakening, which is fatal to the career achievements for athletes ${ }^{[11]}$. The bone-patellar tendon-bone autograft surgery has come around many years, and has been proved to be conducive to the rapid recovery of functionality and moveability due to its unique feats, such as bone-bone healing and effective tunnel-graft integration ${ }^{[12-14]}$. However, this invasive method is very traumatic: a larger surgical incision, a fixed length and a weaker graft than the original ACL, and threatens to induce various complications, including pain in the prepatellar area and patella fracture ${ }^{[15]}$.

Autologous peroneal longus tendon has recently been used as a graft to reconstruct injured ligaments ${ }^{[16-}$ 18]. In contrast with semitendinosus tendon and gracilis tendon, PLT is easier to be taken off for its superficial location and no fiber connection with nearby tendons or deep fascia ${ }^{[18]}$. According to Sholahuddin Rhatomy et al ${ }^{[11]}$, the knee joints reconstructed with PLT and hamstring tendon have similar functional scores one year later, but PLT has the advantages of a larger diameter and a lower incidence of thigh malnutrition.

Graft diameter is one of the most important considerations during an ACL reconstruction surgery on the knee. It has been reported that when the graft thickness is in the 7-9 $\mathrm{mm}$ range, the probability that the reconstructed ACL will be repaired in future declines by 0.82 times for every $0.5 \mathrm{~mm}$ increase in its thickness ${ }^{[19]}$. Studies have shown that when the graft diameter is less than $8 \mathrm{~mm}$, both IKDC and KOOS scores increase for every $1 \mathrm{~mm}$ increase in its diameter ${ }^{[20,21]}$. PLT has a larger diameter, and thus better toughness and strength than other grafts ${ }^{[22]}$. Given peroneus longus plays an important role in assisting foot valgus and plantar flexion, so an important reason why PLT has not been widely used in the reconstruction of clinically injured ligaments is the worry that the absence of PLT will affect the function of the donor ankle joint and cause the ankle instability ${ }^{[23]}$. An earlier study by Angthong et al ${ }^{[11]}$. mentioned that PLT use threatens to induce complications at the donor site, including reduced average peak torque of foot valgus and plantar flexion, and weaken the ankle functionality and stability. However, with the 32 patients we followed up, there was no obvious pain in the ankle joint, no movement limitation, a stable ankle-hind foot, a good foot alignment and an Afos score of 94.7 \pm 6.8 (in the 90-100 range), indicating that the PLT transplantation from the affected side for reconstructing the anterior cruciate ligament has no significant effect on the functionality of the donor ankle joint. Sholahuddin Rhatomy et al $^{[22]}$. used a modified dynamometer to assess ankle joint function. The results showed that Ankle eversion and first ray plantarflexion strength at the donor site were similar to those at the contralateral healthy site, with no donor site morbidity. The reason may be that the functionality of PLT is compensated by PBT, posterior tibial tendon and adjacent muscles. One year after the operation, there 
was no fracture of the graft, which indicates that PLT has enough strength as a graft for ACL. It has been reported in one literature that the distal end of the PLT needs to be sutured with the PBT. However, in our operation, the distal end of the PLT was not braided and sutured to the PBT, and the patient's ankle joint still functions well after the operation. During the follow-ups, 25 of the 32 patients (78.1\%) had symptoms of sore knees on the affected side during rainy days and cold. We suggest that patients after ACL reconstruction should pay attention to keeping the knee joint warm.

This research has certain limitations: First of all, the follow-up time is short, and a longer follow-up time (at least two years) is needed to evaluate the knee joint function after ACL reconstruction; secondly, the amount of data is less. Only 32 eligible patients were included in the analysis. It can be seen that the standard deviation of the data in this Paper is slightly larger, indicating that the data fluctuates greatly and the sample size is smaller; thirdly,only some scales are used to evaluate function, which is highly subjective. Subsequent research can use some instruments to analyze the stability, muscle strength, gait, etc. of the knee and ankle joints, and come to more objective conclusions; Fourthly, only the effects of PLT as a graft to reconstruct ACL were studied, and no comparison with these of other materials was made. Therefore, We cannot explain the advantages of PLT over other materials.

\section{Conclusion}

This research proved that the single-bundled double-stranded PLT transplantation for reconstructing the anterior cruciate ligament can obtain a good knee function recovery and has no obvious side effects on the donor ankle joint. PLT is a good choice as a graft in PCL reconstruction.

\section{Declarations}

\section{Acknowledgements}

We would like to thank Jinshan Hospital affiliated to Fudan University for helping us get in touch with patients.

\section{Ethics approvals and consent to participate}

Approval has been given by the Ethics Committee of Jinshan Hospital, Fudan University (IEC-2020-S06)

All experimental protocols were approved by Ethics Committee of Jinshan Hospital, Fudan University (IEC-2020-S06)

All methods were carried out in accordance with Chinese Orthopaedic Foot \&Ankle Sociaty's guidelines Informed consent was obtained from all participants and all the patients age were over 18 years.

\section{Funding}


Jinshan Hospital Affiliated to Fudan University Young Doctors Research Startup Fund

\section{Availability of data and materials}

Due to Jinshan hospital's regulations, the datasets used during the current study will not be made publicly available. Please contact the corresponding author if necessary.

\section{Competing interests}

The authors declare that they have no competing interests.

\section{Consent for publication}

Not applicable.

\section{Authors' contribution}

ZG, BNW, XCZ, and SCZ were each involved in the design, literature search, model development, data interpretation and writing. BNW conducted the statistical analysis and prepared the first draft. All authors read and approved the final manuscript.

\section{Contributor Information}

ZHE GE, Email: 763144116@qq.com

XINCHAO ZHANG, Email: zhangxc410@aliyun.com

BINGNAN WANG, Email: wbn20130901@163.com

SHICHAO ZHANG, Email: zhangshichao000001@foxmail.com

\section{References}

1. FLOSADOTTIR V, FROBELL R, ROOS E M. Impact of treatment strategy and physical performance on future knee-related self-efficacy in individuals with ACL injury . BMC Musculoskelet Disord. 2018, 19(1): 50. doi: 10.1186/s12891-018-1973-2

2. TAINAKA K, TAKIZAWA T, KOBAYASHI H. Limited hip rotation and non-contact anterior cruciate ligament injury: a case-control study. Knee. 2014, 21(1): 86-90.doi: 10.1016/j.knee.2013.07.006

3. ANGTHONG C, CHERNCHUJIT B, APIVATGAROON A. The Anterior Cruciate Ligament Reconstruction with the Peroneus Longus Tendon: A Biomechanical and Clinical Evaluation of the Donor Ankle Morbidity . J Med Assoc Thai, 2015, 98(6): 555-560.

4. PALMER J E, RUSSELL J P, GRIESHOBER J. A Biomechanical Comparison of Allograft Tendons for Ligament Reconstruction. Am J Sports Med, 2017, 45(3): 701-707. doi: 10.1177/0363546516671944 
5. BI M, ZHAO C, ZHANG S. All-Inside Single-Bundle Reconstruction of the Anterior Cruciate Ligament with the Anterior Half of the Peroneus Longus Tendon Compared to the Semitendinosus Tendon: A Two-Year Follow-Up Study. J Knee Surg, 2018, 31(10): 1022-1030. doi: 10.1055/s-0038-1627466.

6. GRIFFIN L Y, AGEL J, ALBOHM M J. Noncontact anterior cruciate ligament injuries: risk factors and prevention strategies . J Am Acad Orthop Surg, 2000, 8(3): 141-150. doi: 10.5435/00124635200005000-00001.

7. ROTHRAUFF B B, JORGE A, DE SA D. Anatomic ACL reconstruction reduces risk of post-traumatic osteoarthritis: a systematic review with minimum 10-year follow-up. Knee Surg Sports Traumatol Arthrosc, 2020, 28(4): 1072-1084. doi: 10.1007/s00167-019-05665-2

8. SANDERS T L, KREMERS H M, BRYAN A J. Is Anterior Cruciate Ligament Reconstruction Effective in Preventing Secondary Meniscal Tears and Osteoarthritis? . Am J Sports Med, 2016, 44(7): 16991707. doi: $10.1177 / 0363546516634325$.

9. RHATOMY S, HARTOKO L, SETYAWAN R. Single bundle ACL reconstruction with peroneus longus tendon graft: 2-years follow-up. J Clin Orthop Trauma, 2020, 11(Suppl 3): S332-s336. doi: 10.1016/j.jcot.2019.09.004.

10. MOUARBES D, MENETREY J, MAROT V. Anterior Cruciate Ligament Reconstruction: A Systematic Review and Meta-analysis of Outcomes for Quadriceps Tendon Autograft Versus Bone-Patellar Tendon-Bone and Hamstring-Tendon Autografts. Am J Sports Med, 2019, 47(14): 3531-3540. doi: $10.1177 / 0363546518825340$.

11. RHATOMY S, ASIKIN A I Z, WARDANI A E. Peroneus longus autograft can be recommended as a superior graft to hamstring tendon in single-bundle ACL reconstruction. Knee Surg Sports Traumatol Arthrosc, 2019, 27(11): 3552-3559. doi: 10.1007/s00167-019-05455-w.

12. ROMANINI E, D'ANGELO F, DE MASI S. Graft selection in arthroscopic anterior cruciate ligament reconstruction. J Orthop Traumatol, 2010, 11(4): 211-219. doi: 10.1007/s10195-010-0124-9

13. SCHODERBEK R J, JR., TREME G P, MILLER M D. Bone-patella tendon-bone autograft anterior cruciate ligament reconstruction. Clin Sports Med, 2007, 26(4): 525-547. doi: 10.1016/j.csm.2007.06.006.

14. AKOTO R, ALBERS M, BALKE M. ACL reconstruction with quadriceps tendon graft and press-fit fixation versus quadruple hamstring graft and interference screw fixation - a matched pair analysis after one year follow up. BMC Musculoskelet Disord, 2019, 20(1): 109. doi: 10.1186/s12891-0192499-y.

15. SHELTON W R, FAGAN B C. Autografts commonly used in anterior cruciate ligament reconstruction. J Am Acad Orthop Surg, 2011, 19(5): 259-264. doi: 10.5435/00124635-201105000-00003.

16. Saktia M, Biaktoa K T, Usmana M A, Tedjajuwanaa M J, Pasallo P. Predicting the peroneus longus tendon autograft size in ACL reconstruction by using anthropometric parameters: A study in South Sulawesi population. J Orthopaedics, 2020, 22(1-4). doi: 10.1016/j.jor.2020.03.011.

17. TRUNG D T, MANH S L, THANH L N. Preliminary Result of Arthroscopic Anterior Cruciate Ligament Reconstruction Using Anterior Half of Peroneus Longus Tendon Autograft. Open Access Maced J 
Med Sci, 2019, 7(24): 4351-4356. doi: 10.3889/oamjms.2019.390

18. ZHAO J, HUANGFU X. The biomechanical and clinical application of using the anterior half of the peroneus longus tendon as an autograft source. Am J Sports Med, 2012, 40(3): 662-671. doi: $10.1177 / 0363546511428782$.

19. SPRAGG L, CHEN J, MIRZAYAN R. The Effect of Autologous Hamstring Graft Diameter on the Likelihood for Revision of Anterior Cruciate Ligament Reconstruction. Am J Sports Med, 2016, 44(6): 1475-1481. doi: 10.1177/0363546516634011

20. MARISCALCO M W, FLANIGAN D C, MITCHELL J. The influence of hamstring autograft size on patient-reported outcomes and risk of revision after anterior cruciate ligament reconstruction: a Multicenter Orthopaedic Outcomes Network (MOON) Cohort Study. Arthroscopy, 2013, 29(12): 19481953. doi: 10.1016/j.arthro.2013.08.025

21. MOHTADI N G, CHAN D S, DAINTY K N. Patellar tendon versus hamstring tendon autograft for anterior cruciate ligament rupture in adults. Cochrane Database Syst Rev, 2011, 2011(9): Cd005960. doi: 10.1002/14651858.CD005960.pub2.

22. RHATOMY S, WICAKSONO F H, SOEKARNO N R. Eversion and First Ray Plantarflexion Muscle Strength in Anterior Cruciate Ligament Reconstruction Using a Peroneus Longus Tendon Graft. Orthop J Sports Med, 2019, 7(9): 2325967119872462. doi: 10.1177/2325967119872462

23. MURLEY G S, TAN J M, EDWARDS R M. Foot posture is associated with morphometry of the peroneus longus muscle, tibialis anterior tendon, and Achilles tendon. Scand J Med Sci Sports, 2014, 24(3): 535-541. doi: 10.1111/sms.12025.

\section{Tables}

Due to technical limitations, table 1,2,3 is only available as a download in the Supplemental Files section.

\section{Figures}




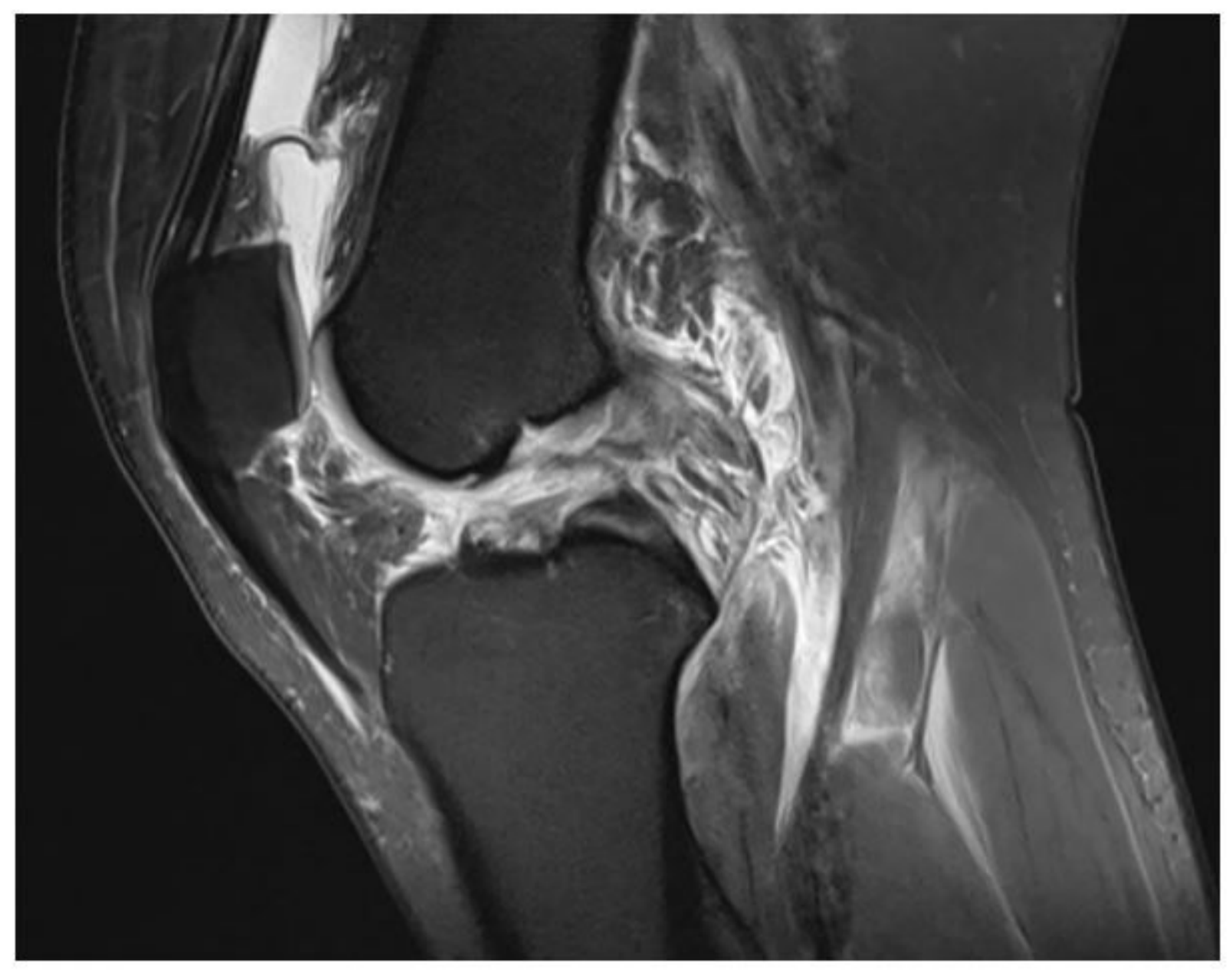

Figure 1

Magnetic resonance imaging revealed complete anterior cruciate ligament (ACL) rupture 


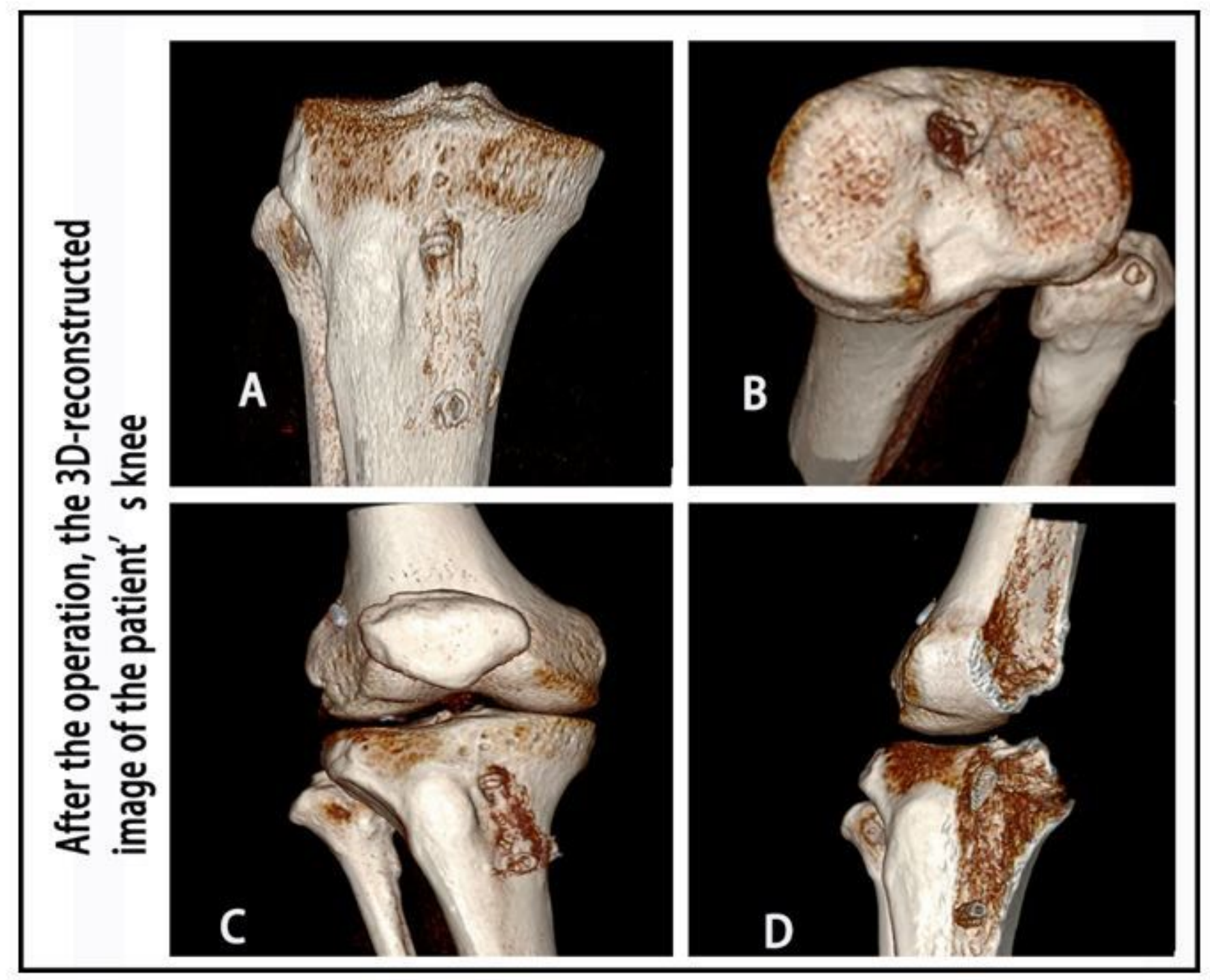

Figure 2

A: The location of the tibial tunnel (Coronal position); B: The location of the tibial tunnel (Cross section) C: The location of the femoral tunnel (Coronal position); D: The location of the femoral tunnel (Sagittal position) 


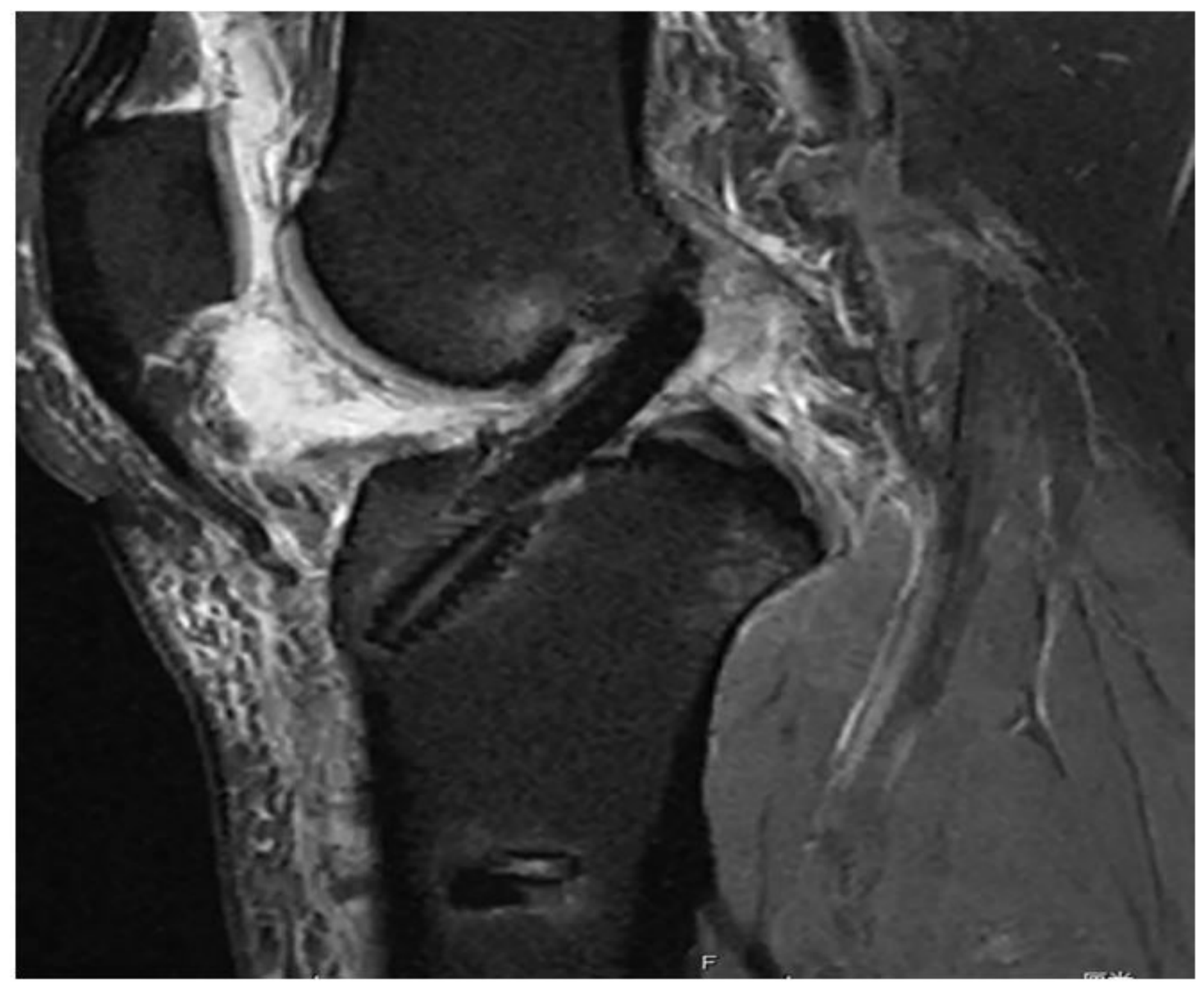

Figure 3

After surgery, MRI showed that the graft was in good position

\section{Supplementary Files}

This is a list of supplementary files associated with this preprint. Click to download.

- Table1.jpg

- Table2.jpg

- Table3.jpg 